LA-UR-01-4885

Approved for public release; distribution is unlimited.

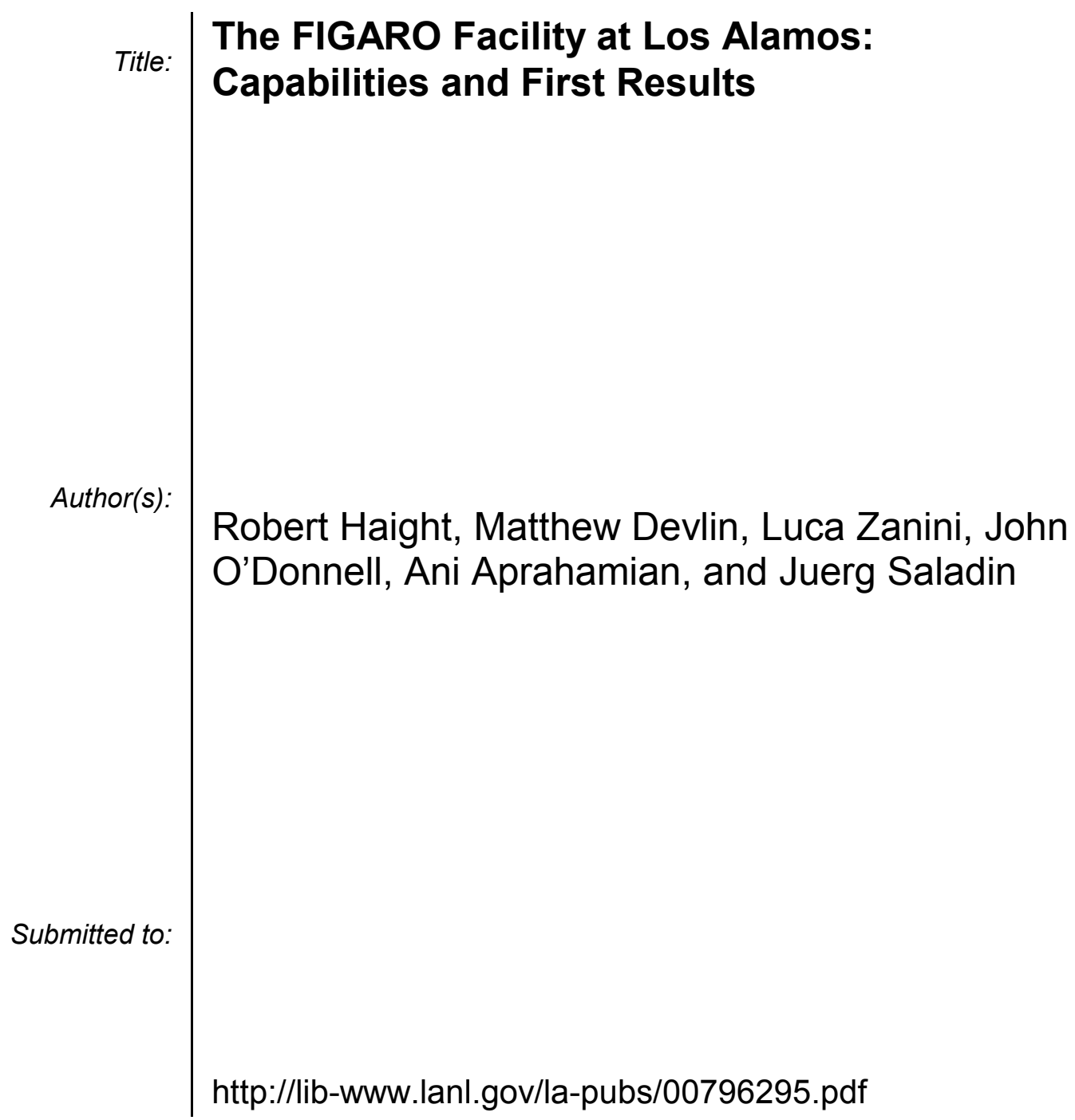

Los Alamos National Laboratory, an affirmative action/equal opportunity employer, is operated by the University of California for the U.S. Department of Energy under contract W-7405-ENG-36. By acceptance of this article, the publisher recognizes that the U.S. Government retains a nonexclusive, royaltyfree license to publish or reproduce the published form of this contribution, or to allow others to do so, for U.S. Government purposes. Los Alamos National Laboratory requests that the publisher identify this article as work performed under the auspices of the U.S. Department of Energy. Los Alamos National Laboratory strongly supports academic freedom and a researcher's right to publish; as an institution, however, the Laboratory does not endorse the viewpoint of a publication or guarantee its technical correctness. 


\title{
The FIGARO Facility at Los Alamos: Capabilities and First Results
}

\author{
Robert HAIGHT ${ }^{1, *}$, Matthew DEVLIN ${ }^{1}$, Luca ZANINI ${ }^{1,2}$, John O’DONNELL ${ }^{1}$, \\ Ani APRAHAMIAN ${ }^{3}$, and Juerg SALADIN ${ }^{4}$ \\ ${ }^{1}$ Los Alamos National Laboratory, Los Alamos, NM 87545, USA \\ ${ }^{2}$ Present address: CERN, Geneva, Switzerland \\ ${ }^{3}$ University of Notre Dame, Notre Dame, IN 46556, USA \\ ${ }^{4}$ University of Pittsburgh, Pittsburgh, PA 15260, USA
}

\begin{abstract}
A new beam line at the fast neutron spallation source at Los Alamos Neutron Science Center has been constructed for studies of neutron-induced reactions producing gamma rays, internal conversion electrons or neutrons. This facility, called FIGARO (Fast neutron-Induced GAmma-Ray Observer), follows on the great successes of GEANIE (described in other contributions to this Conference), by detecting de-excitation gamma rays with high-resolution germanium detectors. FIGARO has fewer gamma-ray detectors than GEANIE, but instead offers other features including: extremely good collimation of the neutron beam for background reduction, a flexible experimental area to optimize detection efficiency and to allow evaluation of other detectors such as ICEBALL-II for internal conversion electrons, inclusion of neutron detectors for the study of neutron-gamma coincidences, beam time to relieve the scheduling pressure on GEANIE, and a PC-based data acquisition system. Our initial measurements include level density studies through ${ }^{59} \mathrm{Co}(\mathrm{n}$,xgamma $)$ reactions to complement our previous ${ }^{59} \mathrm{Co}(\mathrm{n}$,xalpha) measurements, reaction studies of $\mathrm{MeV}$ neutrons on ${ }^{99} \mathrm{Tc}$ with the goal of determining cross sections relevant to transmutation and neutron transport in the design of facilities to incinerate nuclear waste, and an assessment of measuring internal conversion electrons, rather than gamma rays, produced by neutron excitation of actinides.
\end{abstract}

KEYWORDS: neutron-induced reactions, gamma-ray detection, conversion electrons, neutron emission

\section{Introduction}

The interactions of fast neutrons with nuclei continue to be of great interest in both basic and applied nuclear physics. Continuous-in-energy neutron sources from electron linacs and spallation neutron facilities are able to cover wide ranges of neutron energies in a single experiment, and this wide range of energies is crucial in validating nuclear reaction models and in providing cross section data for applied data bases. The study of prompt gamma rays emitted from the neutron-nucleus interactions allows the identification of the residual nuclei and puts reaction models to the test of accounting not only for the observed cross sections but also to the relative strength of the transitions, which are influenced by details in the gamma-ray cascade feeding specific residual levels and by the initial levels populated in the strong interaction (e.g. particle emission from the compound nucleus and excitation in direct or preequilibrium reactions).

At the Los Alamos Neutron Science Center (LANSCE), prompt gamma-ray production has been studied for many years. 1) More recently the GEANIE array of 26 or more high-resolution gamma-ray detectors has proved very useful in reaction studies as well as in the investigation of nuclear structure. This facility is oversubscribed by about a factor of 3 at present. Therefore we needed an additional capability for studying prompt gamma rays.

The FIGARO (Fast neutron-Induced Gamma-Ray Observer) facility was established to enable further measurements of prompt gamma rays. In the design of this facility, we wished to allow as well other types of measurements including neutron emission. The n-gamma coincidence opens the possibility of measuring the neutron emission spectrum gated by a specific gamma ray in the residual nucleus. Schematically, the decay of the compound nucleus with the emission of only one neutron to populate states in the residual nucleus is illustrated in Fig. 1.

Two other types of measurements are possible with this flexible facility. Instead of triggering an event with the gamma-ray detectors a fission chamber can be placed in the beam to study the fission process. The neutron detectors can therefore be used to study neutron-emission in fission. The first attempts at such a measurement are now in progress. The other possibility is to trigger the event on conversion electron spectrometers to focus on electromagnetic transitions that are highly converted. We attempted that last year with the ICEBALL array ${ }^{2)}$ from the University of Pittsburgh.

\footnotetext{
* Corresponding author, Tel. +1-505-667-2829, Fax. +1-505 -6673705, E-mail: haight@lanl.gov.
} 


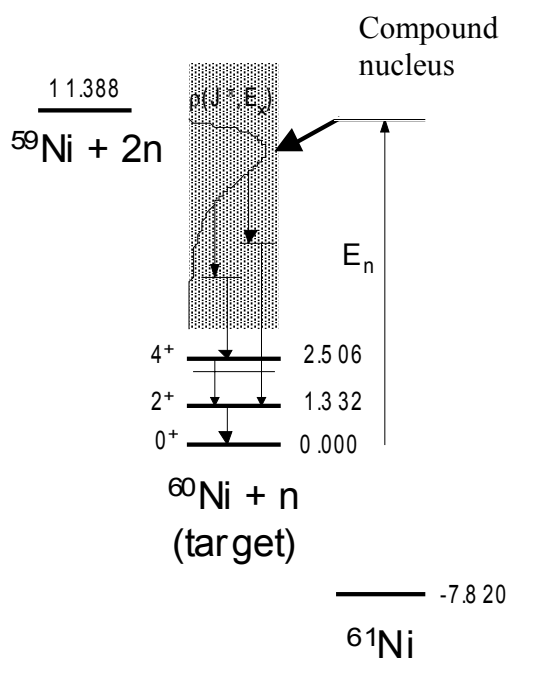

Fig 1. Schematic of the compound nucleus decay with the emission of only one neutron to populate states in the residual nucleus. Low-lying states are populated by gamma-ray cascades from initial states reached by neutron emission.

\section{The FIGARO Facility}

The FIGARO facility is shown schematically in Fig. 2. It has been described briefly elsewhere. ${ }^{3)}$ The WNR beam at the 30-degrees right neutron-production angle ${ }^{4,5}$ passes through a shutter with an adjustable opening up to $10 \mathrm{~cm} \mathrm{x}$ $10 \mathrm{~cm}$, although the shutter is usually opened no more than $2.5 \mathrm{~cm} \times 2.5 \mathrm{~cm}$. Further collimation allows the beam to be used first for charged- particle emission experiments from neutron-induced reactions in another apparatus. ${ }^{6}$ Because this upstream station removes almost no neutrons from the beam, the full flux can then pass through other collimators to the 22 meter station where the samples are placed. At this point the beam is typically $2 \mathrm{~cm}$ in diameter, but it can be made smaller or larger by a different set of collimator inserts. At the FIGARO neutron-production angle of 30 degrees, the neutrons per steradian are similar to GEANIE up to $30 \mathrm{MeV}$, and above that energy there are more neutrons per steradian in FIGARO, which has a factor of 2 more neutrons per steradian at $150 \mathrm{MeV}$.

The neutron fluence is monitored by a fission chamber) mounted 3 meters downstream of the sample position. This location is non-interfering with the collimation just upstream of the sample. Neutrons scattered from the windows of the fission chamber mostly go forward and have no chance to scatter backwards to the germanium detectors. A correction is made for attenuation of the neutron beam by the sample.

The principal detectors for FIGARO are high-resolution HPGe detectors, a coaxial and, for the lower gamma-ray energies, a planar detector. An automated liquid nitrogen filling system keeps these detectors at the correct, very low temperature. The system has been running for about a year with no appreciable degradation of resolution due to neutron damage.

For gamma-neutron coincidences, there is an array of liquid-scintillator neutron detectors located on a circle centered at the sample with a radius of 1 meter. The neutron detector array is at present in development. Shorter or longer flight paths are possible at the expense of time (and energy) resolution or efficiency, respectively. At present six neutron detectors are in place and we expect at least four more very soon. The liquid is NE213 or EJ301 and the detectors are typically $12.5 \mathrm{~cm}$ in diameter and $5.08 \mathrm{~cm}$ thick. With a time-resolution of 5-10 ns for the germanium detectors, the 1-meter flight path gives a neutron energy resolution of 0.14 to $0.28 \mathrm{MeV}$ at $1 \mathrm{MeV}$ and 1.5 to $3.2 \mathrm{MeV}$ at $5 \mathrm{MeV}$. Clearly there is an incentive for improvements in timing of the germanium detectors.

Because of the flexibility of this flight path, other detectors can be used as well. Two types of detector have been used in place of the germanium detector. The first is an array of multi-gap ("orange") conversion electron spectrometers called ICEBALL-II. ${ }^{2)}$ This array is especially useful for studying electromagnetic transitions that are highly converted, such as relatively low energy transitions in nuclides of high atomic number or monopole (E0) transitions, which proceed only by internal conversion.

\section{Figaro}

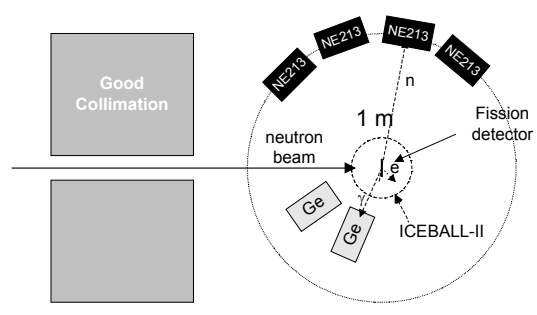

Fig 2. Layout of the FIGARO facility showing the collimation and detectors for gamma rays, neutrons, conversion electrons and fission fragments. The center of the array is approximately 22 meters from the fast neutron spallation source at LANSCE/WNR.

The other type of detector, which can be used in the beam, is a fission detector. By gating on fission events, the neutron emission spectra from fission can be measured as a function of neutron energy over the full range of the neutron spectrum at this flight path. Such measurements are beginning now.

For most of these detector configurations, the same electronics can be used. The signals are processed by standard NIM electronics and are then fed to CAMAC analog-to-digital converters, time digitizers, and scalers. Because of the macropulse structure of the beam, which is on for 625 microseconds followed by 8 milliseconds of down-time, and because of the high instantaneous count rate 
during the beam-on period, we store the digitized data in the CAMAC crate and then read them out between macropulses. The data are read into a personal computer with the MIDAS data acquisition package ${ }^{8)}$ and the results are displayed by ROOT $^{9)}$

\section{Performance of FIGARO}

FIGARO has been used so far in two types of experiments and two more are in development at the time of this meeting. Gamma-ray singles experiments are being carried out in the mass A $\sim 60$ region to complement chargedparticle emission studies ${ }^{3)}$ and in other regions such as with a radioactive ${ }^{99} \mathrm{Tc}$ sample. The measurements with stable isotopes are straightforward and data can be acquired in runs of only a few days.

We attempted measurements of conversion electrons (CE) with ICEBALL-II. These measurements were not successful, but the technique is still very intriguing. The signal was expected to be small because very thin samples (a few $\mathrm{mg} / \mathrm{cm}^{2}$ ) were required so that the electrons could exit the sample with little energy loss. These samples are therefore thinner than used in the gamma-ray singles experiments by a factor of 100 or so. ICEBALL-II does have a higher efficiency for detecting the CE's than the germanium detectors have for detecting gamma rays by a factor of about 10 , and so that leaves a factor of 10 or so for counting rate in favor of the germanium detector experiments, for equal numbers of CEs and gamma rays. The other significant factor is that the silicon detectors in ICEBALL-II were closer to the neutron beam than the typical germanium detector. Background rates were therefore higher in ICEBALL-II. Nevertheless, the possibility of detecting CE would open areas of experimentation unavailable to gammaray detection. Perhaps a superconducting solenoid, which would transport the electrons far from the sample to a low background environment, would be a more successful approach. These experiments with neutrons avoid the problem in charged-particle experiments of backgrounds from delta-ray electrons produced by the charged-particle beam.

We are now measuring neutron emission spectra in coincidence with gamma rays that uniquely identify the residual nuclei. For studies of fission neutron spectra as a function of incident neutron energy, the fission event is detected by an ionization counter in the beam and the emitted neutron spectrum is measured by time of flight to the neutron detectors.

\section{Applications}

The applications of the FIGARO facility include nuclear data measurements of gamma-ray production, inference of channel cross sections from the gamma-ray data (e.g Ref. 1), a significant extension of isomer-ratio-like data to states that decay by prompt gamma-ray emission, nuclear spectroscopy studies, neutron-emission spectra from n-gamma coincidences that also give some information on the distribution of angular momentum in states populated by the reaction, fission neutron spectra as functions of the incident neutron energy, and tests of reaction models.

An example of singles gamma-ray production data is given in Fig. 3, where several gamma-ray production cross sections from neutron interactions on ${ }^{59} \mathrm{Co}$ are displayed as functions of neutron energy. This first study was undertaken because the results could be compared with previous data from Oak Ridge ${ }^{10)}$ and because we had studied the alphaparticle emission from neutron reactions on cobalt. ${ }^{6}$ In general, our results agree well with those from the previous study where the energy ranges overlap. With the LANSCE spallation neutron source, higher neutron energies are more easily studied and we see that the statistical uncertainties of the present results are better than those of Ref. 10 at neutron energies above $15 \mathrm{MeV}$. In comparisons of the data with calculations, using the same reaction model as described in Ref. 6, we find that for most of the gamma rays, the calculations reproduce quite well the observed cross sections. An exception in the case of the ${ }^{59} \mathrm{Co}(\mathrm{n}, 2 \mathrm{n})$ reaction to the $5^{+}{ }_{2}$ state of ${ }^{58} \mathrm{Co}$ at $374 \mathrm{keV}$, which decays by a 320.8 $\mathrm{keV}$ gamma ray. This cross section is over-calculated by a factor of about 2. An outstanding issue is whether this disagreement is due to nuclear structure problems in the gamma-ray cascade feeding the $374 \mathrm{keV}$ state or to some feature of the reaction mechanism.

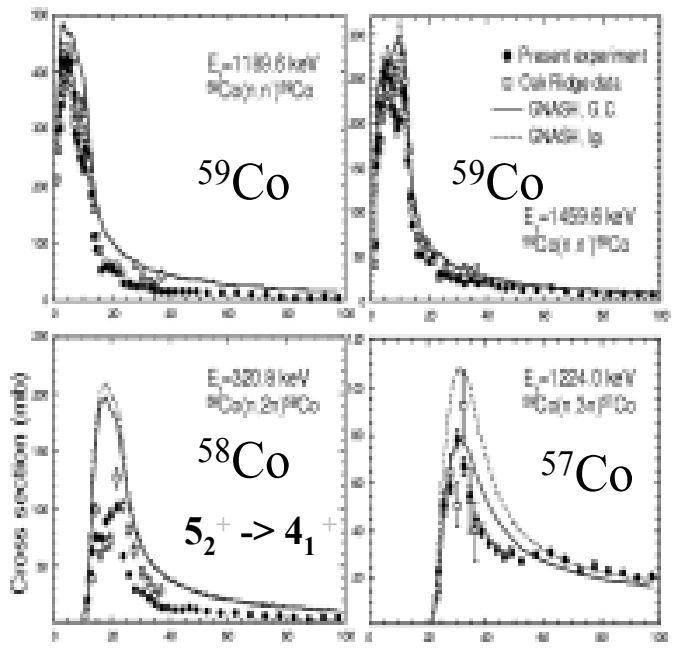

Fig. 3 Excitation functions of 4 gamma rays produced by neutron interactions with cobalt. Reaction model calculations can reproduce most of these data. The calculations use GilbertCameron (G.C.) or Ignatyuk (Ig.) level densities. The Oak Ridge data are from Ref. 10.

These gamma-ray data constitute a significant extension of isomer ratios, which have been a subject of interest and discussion for many years. The present case is complementary to isomer ratios because only states that decay promptly, in a few tens of nanoseconds or faster, can be used with the time-of-flight technique. Isomers on the other hand require relatively long-lived states. Because most levels decay promptly, the present technique opens up the field by a factor determined by the number of gamma-rays that can be detected. Usually this factor is on the order of 
10 times the number of levels ( 2 or 3 usually) accessible by detecting the decay of isomeric levels.

Another example is gamma rays from neutron interactions with ${ }^{99} \mathrm{Tc}$. At lower energies, total and capture cross sections have been measured. ${ }^{11,12)}$ A spectrum integrated over all energies in the present experiment is given in Fig. 4. Many gamma rays from inelastic processes are observed as well as some from $(\mathrm{n}, 2 \mathrm{n})$ and other reactions. These data will be compared with calculations to infer the cross sections for $(n, 2 n)$ and other important reactions.

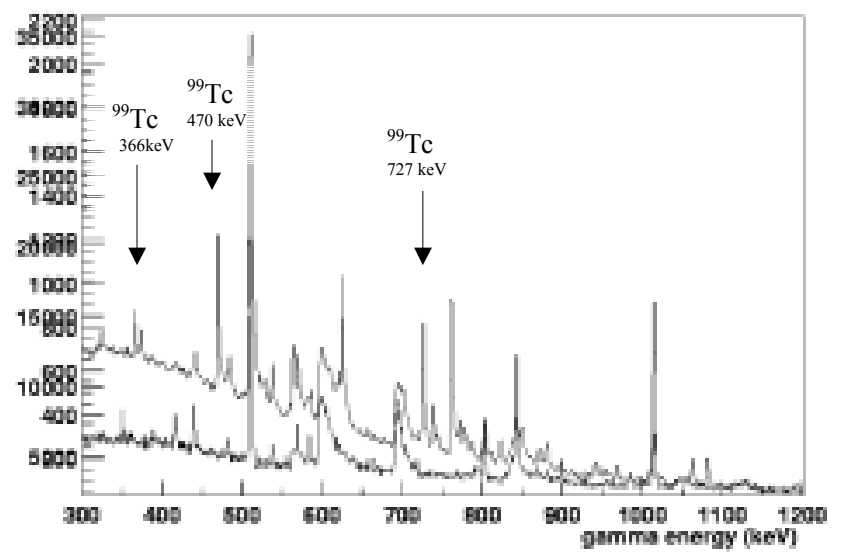

Fig. 4 Spectrum of gamma rays emitted from neutron reactions with the ${ }^{99} \mathrm{Tc}$ sample. The upper spectrum is ${ }^{99} \mathrm{Tc}$ plus the containment can; the lower spectrum (normalized) is from an empty can. The assignments for a few typical lines are indicated.

\section{Conclusions}

FIGARO constitutes a significant addition to the capabilities at LANSCE to measure prompt gamma rays and to study reactions where neutron emission spectra are of interest. It is flexible and, so far, has accommodated not only high-resolution gamma-ray detectors, but also conversion electron spectrometers and fission detectors. This flexibility complements the capabilities of GEANIE. Nuclear data for basic and applied programs are being obtained with this new facility.

\section{Acknowledgment}

This work has benefited from the use of the Los Alamos Neutron Science Center at the Los Alamos National Laboratory. This facility is funded by the US Department of Energy and operated by the University of California under Contract W-7405-ENG-36.

\section{References}

1) H. Vonach, A. Pavlik, M. B. Chadwick, R. C. Haight, R. O. Nelson, S. A. Wender, and P. G. Young, ${ }^{207,208} \mathrm{~Pb}(\mathrm{n}, \mathrm{xn} \gamma)$ Reactions for Neutron Energies from 3 to $200 \mathrm{MeV}, "$ Phys. Rev., C50, 1952 (1994).
2) M. P. Metlay, J. X. Saladin, I. Y. Lee, O. Dietzsch, "The ICEBall: a multiple-element array for in-beam internal conversion electron spectroscopy," Nucl. Intr. Methods, A 336, 162 (1993).

3) L. Zanini, A. Aprahamian, M. B. Chadwick, M. Devlin, R. C. Haight, J. X. Saladin, and P. G. Young, "Study of Reactions of Fast Neutrons with Nuclei with FIGARO at LANSCE," Proc. $16^{\text {th }}$ International Conference on the Application of Accelerators in Research and Industry (CAARI2000), November 1-4, 2000, Denton, TX. pp . 346-349.

4) P. W. Lisowski, C. D. Bowman, G. J. Russell, and S. A. Wender, "The Los Alamos National Laboratory Spallation Neutron Sources," Nucl. Science and Eng., 106, 208 (1990).

5) H. Condé, R. Haight, H. Klein and P. Lisowski, "New Neutron Facilities for Nuclear Data Measurements at $\mathrm{E}_{\mathrm{n}}$ $>10 \mathrm{MeV}$," Proc. Int. Conf. on Nuclear Data for Science and Technology, Jülich 13-17 May, 1991, ed. S. M. Qaim, (Springer-Verlag, Berlin 1992) p. 386.

6) S. M. Grimes, C. E. Brient, F. C. Goeckner, F. B. Bateman, M. B. Chadwick, R. C. Haight, T. M. Lee, S. M. Sterbenz, P. G. Young, O. A. Wasson, and H. Vonach, "The ${ }^{59} \mathrm{Co}(\mathrm{n}, \alpha)$ Reaction from 5 to $50 \mathrm{MeV}$," Nucl. Sci. Eng., 124, 271 (1996).

7) S. A. Wender, S. Balestrini, A. Brown, R. C. Haight, C. M. Laymon, T. M. Lee, P. W. Lisowski, W. McCorkle, R. O. Nelson, W. Parker, and N. W. Hill, "A Fission Ionization Detector for Neutron Flux Measurements at a Spallation Source," Nucl. Instr. Meth. in Physics Res., A336, 226 (1993).

8) Stefan Ritt and Pierre-André Amaudruz, "MIDAS, Maximum Integrated Data Acquisition System," Paul Scherrer Institut and TRIUMF, http://midas.triumf.ca/ (2001).

9) Rene Brun and Fons Rademakers, "ROOT - An Object Oriented Data Analysis Framework," Proceedings AIHENP'96 Workshop, Lausanne, Sept. 1996, Nucl. Inst. Meth. in Phys. Res., A 389 (1997) 81-86. See also http://root.cern.ch.

10) T. M. Slusarchyk, "Preliminary cross sections for gamma rays produced by interaction of 1 and $40 \mathrm{MeV}$ neutrons with ${ }^{59}$ Co," ORNL/TM-11404, Oak Ridge National Laboratory (1989).

11)C. Raepsaet, C. Bastian, F. Corvi, F. Gunsing, and A. Leprêtre, "Measurement of the Neutron Capture Cross Section of ${ }^{99} \mathrm{Tc}$ in the Energy Range from 3 to $400 \mathrm{eV}$," Proc. Int. Conf. Nucl. Data for Science and Technology, Trieste, Italy, May 18-24, 1997, (LA-UR-97-1791), ed. G. Reffo, A. Ventura, and C. Grandi (Italian Physical Society, Bologna, Italy, 1997), pp. 1289-1292.

12) F. Gunsing, A. Brusegan, A. Leprêtre, C. Mounier, and C. Raepsaet, Measurement of the Neutron Total Cross Section of ${ }^{99}$ Tc in the Energy Range from 3 to $600 \mathrm{eV}$," Proc. Int. Conf. Nucl. Data for Science and Technology, Trieste, Italy, May 18-24, 1997, (LA-UR-97-1791), ed. G. Reffo, A. Ventura, and C. Grandi (Italian Physical Society, Bologna, Italy, 1997), pp. 1293-1296. 